\title{
The Ethics of Driverless Cars
}

\author{
Neil McBride \\ Centre for Computing and Social Responsibility \\ De Montfort University, \\ The Gateway, Leicester \\ LE1 9BH \\ Email:nkm@.dmu.ac.uk
}

\begin{abstract}
This paper critiques the idea of full autonomy, as illustrated by Oxford University's Robotcar. A fully autonomous driverless car relies on no external inputs, including GPS and solely learns from its environment using learning algorithms. These cars decide when they drive, learn from human drivers and bid for insurance in real time. Full autonomy is pitched as a good end in itself, fixing human inadequacies and creating safety and certainty by the elimination of human involvement. Using the ACTIVE ethics framework, an ethical response to the fully autonomous driverless cars is developed by addressing autonomy, community, transparency, identity, value and empathy. I suggest that the pursuit of full autonomy does not recognise the essential importance of interdependencies between humans and machines. The removal of human involvement should require the driverless car to be more connected with its environment, drawing all the information it can from infrastructure, internet and other road users. This requires a systemic view, which addresses systems and relationships, which recognises the place of driverless cars in a connected system, which is open to the study of complex relationships, both networked and hierarchical.
\end{abstract}

\section{Categories and Subject Descriptors}

K.4.1 [Computers and Society]: Public Policy Issues: Ethics

\section{General Terms}

Human Factors

\section{Keywords}

Driverless cars, ethics, full autonomy.

\section{INTRODUCTION}

As traffic congestion increases, pollution from traffic, particularly in cities becomes more of an issue and accidents

Permission to make digital or hard copies of part or all of this work for personal or classroom use is granted without fee provided that copies are not made or distributed for profit or commercial advantage, and that copies bear this notice and the full citation on the first page. Copyrights for thirdparty components of this work must be honored. For all other uses, contact the owner/author(s). Copyright is held by the author/owner(s). caused by cars kills millions, there is a need for changing how transport is managed and applying ICT and intelligent systems to providing and managing transport. This requires many systems, connecting networks of systems, understanding how communities work, developing and managing systems within smart cities. Smart transport is community based. It relies on a balance of autonomy, cooperation and regulation. It involves an exchange of knowledge between experts and users; linked systems, addressing choice, flexibility and safety. It involves cooperation with hierarchical systems without compromising freedom and democratic rights. It involves quantitative analysis combined with qualitative perception of people's attitudes, their fear and worries, and an empathic listening to the needs of transportation users.

One component of smart transportation will be driverless cars, operating within cooperative networks, relieving drivers of effort, communicating with each other, and taking into account context and conditions. Interest and investment in connected and driverless cars has grown rapidly. For example, the UK Government are investing $£ 200$ million in research and development into driverless cars. A KPMG report suggested that connected and driverless cars will create 320,000 jobs in the UK. John Leech, Head of Automotive for KPMG, commented that: "Connected and driverless cars will reduce pollution, save lives and promote social inclusion. We owe it to everyone to make this future a reality, " $[4\}$.

The technology for autonomous cars, which drive themselves is developing rapidly. Driverless vehicles could rapidly become common sights on road. Such driverless cars will internally integrate many different inputs to analyse their environments and issues such as road conditions in order to make decisions about where and when to drive. Using learning algorithms, they will build knowledge bases of road conditions and learn to managing unusual and exceptional conditions such as plastic bags blowing across the road, or obstructions in the road [7].

As well as having integrated systems and sensors internally, they may draw on GPS to navigate, or communicate with road traffic systems such as traffic lights, and with other autonomous vehicles, to make decisions about overtaking and to form road train. Autonomous cars may report standard data to central hubs, rather like an aircraft sends out standard data. Location data will enable it to plan routes and change routes as it is progressing. These robot cars will be able to manage 
traffic flow through communication and effectively selforganise traffic systems. Both vehicle to vehicle (V2V) and Vehicle to infrastructure (V2I) may become standard parts of autonomous cars.

The networked, driverless car could be centrally controlled, delivered to a specific location and then programmed to take a fixed route. It can depend on GPS navigation, it can be part of a transport infrastructure, subject to control signals from the infrastructure. It could be constantly monitored and open to intervention from traffic controllers and police, for example, as well as the driver.

These cars may decide when driving conditions are unsuitable. They will learn to improve at driving; they will bid for insurance in real time. Where two cars are involved in a collision, active buckling control may activated so that the cars share information in order to determine who take the brunt of the collision, who buckles most based on size of the car and other factors. The driverless car will offer the driver freedom to sit back, or complete work and to save time. Driving may become a thing of the past. Commuting may become a chance to hold meetings, finish documents.

While many commentators refer to connected cars, some projects are pursuing the development of autonomous driverless cars which are self-contained and have no dependency on anything external to their own capability. These cars will act without a need to consult controllers or satellite navigation systems, independent of infrastructure, not requiring to communicate with other cars, whether driverless or not. All the information the car uses to drive it has learnt for itself. It is an individual, standing alone, driving to its own agenda, neither transmitting information nor needing to receive information. Such a truly autonomous car is a selfcontained system, relying on the information it derives from its own sensors and the learning algorithms built into its onboard computer systems.

Such cars offer the possibility of complete autonomy for the car, which is in effect a robot which can navigate and make its own decisions about when and how to drive as well as how to get to a location. The ambition for autonomous cars is a complete autonomy which does not rely on infrastructure, connection with central systems or even with GPS, an autonomy where the human is eliminated from the loop, an autonomy where the car is self-determining, self-correcting, eventually self-healing and perhaps ultimately self-aware.

This ultimate autonomy may not only prove to be undesirable, but ultimately unachievable. Perhaps born of a philosophy which sees the individual as the paramount object of focus. The truly autonomous car is an enlightened car truly able to think for itself, to employ and rely on its own capabilities to determine what to believe about its environment and how to act. Technological advances and a reliance on the firm ground of mathematics will release it from its self-incurred immaturity; from its inability to use its own understanding without the guidance of another.
Using Oxford University's Robotcar as an example, this paper critiques the philosophy behind autonomous cars with the intention of working towards an inclusive, community ethics which recognises that the deployment and use of autonomous cars is with the context of community and culture and should not be considered as a isolate individual ethics outside the system and relationships within which it operates.

Firstly, the properties of Oxford University's RobotCar are examined and some ethical issues raised. The philosophy and assumptions behind the Robotcar are critiqued with reference to a talk by Paul Newman, the leader of Oxford University's programme in an Intelligence 2 debate in 2013 [5]. Finally, the concepts of an autonomous car and the ethics are considered using the ACTIVE ethics framework and conclusions are drawn.

While autonomous cars offer great benefits, it is important to recognise the limits of self-reliance and that the role of an autonomous car should be considered in the context of the complex social systems and communities within which it operates.

\section{OXFORD UNIVERSITY'S ROBOTCAR}

Robotcar is a modified Nissan Leaf with cameras and laser sensors. It has fly-by-wire control for all aspects including steering wheel, indicators and brakes. It uses a pair of stereo cameras to navigate and lasers to assess $3 \mathrm{D}$ structure of the environment. It will stop if a pedestrian walks in front of it.

\begin{abstract}
Oxford's autonomous vehicle tech does not rely on GPS to navigate because GPS doesn't work well in built-up environments and is in any case not precise or reliable enough to give an exact location. Neither does the Oxford approach use embedded infrastructure such as beacons and guide wires, which often guides robots in factories, as this would be impractical and far too expensive for use in most environments.
\end{abstract}

Instead the Oxford approach to navigation uses algorithms that combine machine learning and probabilistic inference to build up maps of the world around it using data form on-board sensors and 'learn as it drives'. The maps it builds (and updates) are like memories of a route which can be accessed to allow the vehicle to guide itself through places it has been before.[8].

A vehicle controlling computer runs the car, in collaboration with an interface computer and an iPAD. The autonomy is based on a localisation system which uses probability and estimation algorithms to learn about its driver and the environment it is working in. It builds up memories of past historic experiences to refer to. This experience-based navigation uses graphs (path memories) to represent the experience and various algorithms to optimise the time- 
constrained localisation and overcome the problem that as the robot's memories build up, processing takes longer and the robot becomes slower to react.

Hence Robotcar is almost totally self-reliant (although it can carry out on-the-fly internet queries.) This is the goal of total autonomy. In the Robotcar case, the ambition is using available technology to create " $£ 500$ autonomy"

\section{THE PHILOSOPHY OF ROBOTCAR}

What is the philosophy behind Robotcar? What are the goals, the telos of its developers? The head of Robotcar, Professor Paul Newman has given many talks on the use of modern robotics in smart transport, particularly one at a debate on Smarter Mobility in 2013 [5]. I will comment on this talk and other inputs from Newman.

The rhetoric of the Robotcar is one of machine as a response to human inadequacy. Cars cause congestion, accidents and time wasting. They are inefficient. They maim and destroy. This is not because of the machine itself, but the humans who are inadequate. Robotcar involves the elimination of humans from driving because "machines are better at doing stuff than humans." Robotics will fix human inadequacy by replacing humans: Let the cars drive; let the cars bid for insurance in real time; let the cars decide when they can drive; Let the cars get better at driving over time.

Newman draws on a range of reasons why driverless cars are important, particularly with infrastructure-free navigation and full or restricted full autonomy [1]. Firstly there is the rhetoric of safety. Safety is often used as a reason from reducing human interaction and promoting machines. This has been a strong argument for personal health monitors and controlling smart houses. Safety may mean that the designers take over and create functionality which may not be in the user's interest, or required by the user. For example, Google's car intentionally goes over the speed limit to match expected behaviour of other road users. If not known about, this may be deceptive. Hence safety is used as a reason for imposing more control on the human. Secondly, it can be argued that humans are enslaved by cars and that driverless cars free the user. Setting the slave free. Equally it could be argued that driverless cars create a new slavery. The loss of control and interaction is disempowering, deskilling the driver, leaving the driver without knowledge, skills, or the ability to navigate. The occupant of the driverless car, formerly a driver or passenger becomes an object to be moved around from place to place, controlled, moved from A to B efficiently.

Having a goal of full autonomy assumes that self-reliance is good. With no infrastructure, no reliance on authorities, road systems, or other drivers this is real autonomy, an autonomy in which the individual stands alone, independent, the sole arbiter. This may be a working out of an enlightenment view that everyone makes their own decisions without reference to others, without negotiation and outside society or communities. The car will offer the driver autonomy. But who has the autonomy, and who has had control taken from them? This autonomy could be interpreted as a loss of autonomy, a handing of control to the machine. It is the machine that is autonomous and the human that is disempowered. Autonomy is viewed black and white rather than a progression, a negotiation of interdependency between the supporting machine and the supported human.
We also see the rhetoric of "saving precious time". Time is seen as a commodity. We do not ask about the value of that time. Do we replace driving by playing video games? Saving time as a quantitative good may not mean we are gaining anything of human and moral value. And may not reference relationships. For example, driving with my son creates a nonthreatening environment where he may open up and we have useful discussions.

Newman revels in the possibility of an autonomy arms race. Autonomous functions will evolve as manufacturers develop new functions in cars. Behind this perhaps is a war? A battle for power and control between the machine and the human?

Newman treats the autonomy of cars as an inevitable outcome, a sole good and a philosophical end in itself. Indeed, in his talk he pitched this as a belief: "If you don't believe this you need to leave .. this has to be a true thing.'"

\section{THE TECHNOLOGICAL UTOPIA AND AUTONOMOUS CARS}

The utopian position for autonomous cars is one that removes any reliance or connection with outside humans or technology. Even the use of satellite navigation is frowned upon. The ultimate robotcar will rely on the supremacy of the algorithm, as the sole source of truth, a truth that is amenable to logical analysis and proof.

Technology is seen as invincible, provable, permanent, materially-grounded, and reliant only on the solidity of physical laws and mathematics. It is clean, amoral, invulnerable, repeatable, unstained. The only threat of compromise and failure comes from humans included in the loop. Therefore our ultimate goal is the complete exclusion of the humans and the full autonomy of the technology.

The technological utopia contrast the reliability with the fallibility of the human. They are vulnerable, flawed, pathetically unreliable and dangerous. They harbour messiness, uncertainty, unpredictable emotions. Their unpredictability defies mathematical modelling. They cannot be trusted, freed, allocated responsibility because they will inevitably mess things up.

In the technological utopia, the technology absorbs the human. And where problems occur they point out the inadequacy of partial autonomy. Full autonomy does not recognise the interdependency of the machine and the human.

\section{AN ACTIVE ETHICS RESPONSE}

I will examine an ethical response to Robotcar using the ACTIVE ethics framework [6].

\subsection{Autonomy}

For autonomous cars, an important concern is the balance of control between the human and the robot car and the negotiation and transfer of that control based on environmental conditions. There is never total autonomy, the robotcar is an artefact encoding the perceptions of the environment held by the robot engineers. Power and control may be handed to the algorithm developers who decide the rules encoded in the programme. Autonomy is always balanced across an interface between the human and machine. 
The goal of total autonomy may be a goal of deontological disconnection, and ethics in which relationship disappears and the individual is the ultimate arbitrator.

Total autonomy seeks to remove all threats by eliminating any dependency on the outside. If I am self-contained, I'm invulnerable. I have the inner certainty of provable reliable algorithms, rationally supported by the laws of mathematics. Uncertainty, risk and failure is eliminated. I do not even risk a dependency on GPS, which is considered unreliable in builtup areas. The messiness and unpredictability of the human is eliminated from the system. Total autonomy removes the human from the loop: I am logically invulnerable.

And yet the very act of moving on a road in a material world will create vulnerability and risk. My model of a rational, controlled world breaks down the moment I interact with it. There is a leakage into the machine which compromises the total autonomy.

If I elect to learn from the world I only accelerate the leakage by importing unpredictability and messiness into my autonomous system. Robotcar will be expected to learn the style and culture of driving of its host. It could well learn reckless and bad driving. If Robotcar learns the driving and cultural style of its host it simply reproduces the irrationality of the host and renders the pursuit of autonomy pointless/

So total autonomy is sterile, only actioned by deciding to eliminate interaction with the human world and minimise or restrict interaction with the material world. The autonomous system is soon rendered helpless and impotent since any interaction with the surrounding environment involved uncertainty and unpredictability and compromises autonomy. The range of options must narrow and narrow until the only reliable interaction for an autonomous system is with itself or another autonomous system which exactly reproduces its behaviour and is therefore completely predictable. All behaviour outside the autonomous system which does not conform to expectations must be ignores, discarded or eliminated.

It seems to me that total autonomy is not only unachievable but undesirable. The ambition of autonomous systems developers should be focussed on the human / robot interface, the exchanges at the interface and the balance of autonomy and control.

The quality and acceptability of a driverless car may depend not on its withdrawal from the environment and its selfcontainment, but on the capability of the car to interact with its environment and the richness and depth of the interactions which take place with its environment. For the developer, the focus should be on the development of communication, and the capability of rich interaction with the natural, technical and infrastructure systems.

\subsection{Community}

Driverless cars are created out of the interactions of a community, supported by a community of workers and serve a community. They are elements of a community, both as a participants in a relationship between humans and technology and as a technological mediators in social relationships. These cars will depend on a wide range of human interactions and human systems. Communities of cleaners, mechanics, managers, monitors and surveillance staff will support their day-to-day running. Large supplier chains will provide parts, servicing, training for maintainers and regulators. Energy suppliers and the public servants regulating practice will play significant roles. The driverless cars will be extremely dependent of the human communities which will be required to put them on the road and keep them there.

Autonomous cars will exist in a dynamic community. The pedestrians, shop keepers, police, traffic wardens will all interact with driverless cars. The technology connects the community. It cannot exist in desert-island-like isolation. Without community, we can end up with a private transportation hell, where totally autonomous cars compete for parking spaces and clog city centres. Community is about negotiation and compromise: human to human, human to machine, machine to machine.

The suspension of human control in the car should require not less connection and isolation but rather much more connection. In the absence of human intervention a driverless car should seek connection with navigation and advice systems such as weather systems, traffic infrastructure systems, signalling systems, other cars, central control systems, manufacturers' web sites and so on. Every type of connection should be pursued to compensate for the loss of human connection. I do not believe that the human social interaction and environmental awareness can be replaced by learning algorithms. Dialogue with the physical and human environment should be amplified not suppressed in a driverless car. The transfer of competency to the technology requires engagement with a wider knowledge base, not the exclusion of external information.

Driverless cars will. Of course, be subject to risks from security breaches, hacking and the compromising of privacy. However, the solution to the cybersecurity problem associated with autonomous vehicles is not to isolate oneself in total autonomy, to shut oneself down, but to open up and create strong communities of support, knowledge and cooperation to resist the threats.

\subsection{Transparency}

Transparency is a prerequisite for ethical engagement in the development of autonomous cars. There can be nothing hidden, no-cover-ups, no withholding of information. The limits of the driverless car, how it works and how it should be used should be made completely clear. Issues concerning safety, ethical decision making and the setting of boundaries cannot be addressed without transparency. There can be no deception, and no case of the robot car pretending to be what it isn't, creating an illusion of a capability it does not have. There is a difference between imitating a competence and actually having that competence.

The behaviour of Robotcar will depend on the learning algorithms. In the case of personal health monitors, the different algorithms used by the manufactures to turn electrical signals from sensors into data concerning number of steps and distance travelled can result is widely differing figures. And furthermore the meaning of those figures must then be determined. In another example, algorithms for turning the sequences of many short fragments of DNA into genome sequences can vary significantly in their results. The assemblathon competition [2] pits algorithms against one another to see which can come closest to giving the accepted sequence for a benchmark genome. A learning algorithm may varying in its learning and hence is response to environmental stimuli. 
Hence clarity and openness about the algorithms used, how they work and their limitations is not only required technically, but must be communicated in a useful and appropriate way to users, managers, regulators and other interested parties. Limits in data localisation and interpretation need to be understood. The user of an autonomous car needs to be an intelligent user, knowing when to intervene, working with a human-centred interface.

Transparency may require a driving test for driverless cars to demonstrate their competency in navigation, dealing with roads and adhering to codes and laws in the particular geography and culture it will operate in. If we require testing of humans, we should require testing of robot cars.

\subsection{Identity}

In the cartoon film, Wall-E, the rule of technology renders humans passive, incompetent and hedonistic. Free of risk, responsibility and activity, they fail to engage with their environment, to question it and shape their future. They are willing, passive participants in an endless present. Obese, ignorant and unquestioning, they lounge by the swimming pool day after day. They surrender autonomy and responsibility to the technology, computer systems and robots. Additionally they had surrendered their identity. It is only in rebellion, catalysed by Wall-E, that they regain identity and purpose and return the technology to its rightful place. Taking on responsibility and embracing risk, they return to earth to start recolonisation.

Cars are often part of a person's identity. Not only the make and nature of the car, but the competencies in driving and the freedom and control the car provides, constitute part of the person's identity. The removal of competencies by the autonomous vehicle will clearly affect people's identity. In societies where reputation, wealth, and role in society are represented in the car and its use, driverless cars will pose a threat. Resistance to driverless cars may be partly driven by a fear of loss of identity. Social and personal identity may be undermined, or at the least transformed, by the driverless car.

Driverless cars may trigger identity crisis where the person is uncertain about his worth because his skills are transferred to the car. In a sense, by learning to imitate the driving skills and style of the human driver, the car is stealing part of the human's identity and becoming that person by imitating skills and roles which are part of who that person is.

Cars become part of people, extensions of them, gloves to fit into. The car fits the person, and is absorbed into the person. Stripping the person of such connection and involvement with the technology may not result in freedom but a fracturing of the person's identity which leaves them suspended in fear and uncertainty. Conversely for a disabled person, the driverless car may offer an extension of ability. The freedom which results from the ownership and use of a driverless car becomes an important part of that person's identity.

Will the autonomous car make people stupid? Will it steal a person's identity, taking on the driver's personality and characteristics? Understanding the role of the robotcar in human identity will required empathic reflection as well as an investigation of people's perceptions of the role of cars in their lives. People connect with cars. Controlling a car may be seen as a form of freedom. Loss of this human autonomy may equate to loss of identity.

\subsection{Value}

In discussing value we are interested in what people value. Value does not necessarily equate to benefits; it is not about cost benefit. Neither is it about values, our underlying moral drivers. Values will affect what we value. And an analysis of what we value will point to the values underneath. Freedom might be valued above safety, pleasure above health.

In the case of driverless cars, a concern will be on the value we put on the life, the needs and concerns of the users of the robotcar. Do we value external requirements of economics, of efficiency above internal value of promoting human flourishing and excellence? Do we value the driverless car as a statement of technological advance? Are we focussing on the system and the economics over quality of life, or promoting the market and the individual over community and cooperation?

There is a danger of devaluing the driver who becomes an object to be moved around, a set of inputs for the car learning to take over. In considering the ethics of driverless cars, we must also address the need to protect the privacy of information about how the driver drives a car and where and when.

\subsection{Empathy}

"If you don't believe this you need to leave .. this has to be a true thing." Paul Newman, Oxford University [5]

In contrast to impinging our view on the users of autonomous vehicles, an empathic stance requires that we view the deployment and use of the Robotcar through the eyes of the users. This requires us to cross the empathy gap, to put our feet in the shoes of the person sitting in the driverless car. A brief poll of family and friends will reveal a wide range of reactions to a driverless car. Some regard it with fear and revulsion. Wary enough of driven cars and the danger of the roads, the prospect of a driverless car is completely unacceptable. Others may view driverless as a novelty, and want to know 'how it works' out of interest or a need for assurance about the reliability of the technology. The latter point relates to a need for transparency and a reluctance to treat a driverless car as a black box initially and get into it without the sufficient knowledge as to its technology and its reliability.

For some males, the prospect of being driven around by a driverless car may bring about a primitive sense of emasculation.

However viewed, the driverless car elicit emotions and reactions which the engineer must be sensitive to. The engineer has to consider the fears and hopes of drivers; the way of thinking of drivers. There must be a respect for the human. Not treating them as a dangerous annoyance to be removed from the system.

Far from a disconnection with the human and the elimination of the human from the system, empathy requires an increased engagement with the human both in development and deployment. There must be a search for the human behind the driverless cars; a mindfulness of every person experiencing 
the phenomenon, everyone connected with the products, services, infrastructure and usage. There must be a radical listening to the feelings and needs of the user which seeks not to impose a scientific absolute but to respond and adapt to the human heart, and to demonstrate responsiveness and adaptability. There must be an empathy with the perception of risk and vulnerability which new technology elicits. There must be a continuous conversation with the users which determines to reduce the empathy gap.

Questions such as the following should be a matter for reflection and investigation: What is the effect of loss of control, fear of car? What is the effect on the non-user? On those exposed to the car from other cars?

\section{FINDING THE ETHICAL ROLE FOR DRIVERLESS CARS}

Understanding the ethical context of a driverless car requires a systemic understanding of its place as part of the connectivity of transport and indeed society. Autonomy, as mentioned is not an ultimate goal. The driverless car supports and mediates relationships in the community. By enabling a journey for an elderly person, the car should enable connection. Rather than stripping away the autonomy of the driver, stealing his freedom and rendering him a passive recipient, the role of this technology should be seen as that of a support worker, compensating for some frailty of the human, where compensation is appropriate, enabling the human to use of car when physical or social constraints may have prohibited it

The role of support worker does not eliminate risk and vulnerability. Rather it may create new risks and new dependencies. The value of the driverless car will be found not in environmental savings, efficiencies, the "saving of time", and the avoidance of accidents, but rather in the extent that it promotes relationality. It as Coeckelbergh suggests, the ultimate danger is non-relationality [3, p55] then the real ethical worth is in how the driverless car enables people to connect, strengthens communities, enables meetings and human interaction which might have been difficult or impossible before. The ethics of driverless cars is then an ethics of relationship and the impact of the driverless car on the human - human and human- machine relationship. And the key point in relationships is the interface, the boundary at which information is exchanged, understanding achieved, tasks agreed and roles carried out. Using driverless cars will be a matter of teamwork, of working together in the pursuit of common goals and purpose. The robotcar is a connected element in a connected universe, one element connecting to the whole of transport, working with rather than dismissing smart infrastructures, training, and so on. Contributing to the whole, reflecting our dependencies on each other.

\section{CONCLUSION}

Full autonomy is not only practically pointless, it is ethically pointless. The pursuit of such autonomy does not recognise the essential importance of interdependencies between humans and machines and that it is not a case of one or the other, but both. Indeed the splitting of human and machine, of what is perceived as uncertain and risky from the scientific, the assured, the provable, the separation of the rational and the emotional or even the material and the spiritual is a false dichotomy. The technological and the human are more entangled, impossible to prise apart and must be considered as a whole.

This requires a systemic view, which addresses systems and relationships, which recognises the place of driverless cars in a connected system, which is open to the study of complex relationships, both networked and hierarchical, which may give rise to emergent behaviour, and to physical, social and ethical issues which may be unexpected. Gaining an understanding of this will require the development of ethical dialogues between systems, communities and technology. Fundamentally, this requires a human-centred approach, a team approach, which examines the interdependencies between driverless cars and their users.

The pursuit of full robot autonomy is not a practical necessary nor a useful response to our needs and concerns; rather it is born of a philosophical view, underpinned by a particular perception of the human state.

\section{REFERENCES}

[1] Akerman, E. (2013) UK unveils affordable self-driving robot car. IEEE Spectrum. 19 $9^{\text {th }}$ Feb 2013. http://spectrum.iee.org/automaton/robotics/artificialintelligence/uk-affordable-self-driving-robotcar/

[2] Bradnam, K.R. et al (2013) Assemblathon 2: evaluating de novo methods of genome assembly in three vertebrate species. GigaScience, 2:10

[3] Coeckelbergh,M. (2013) Human Being @ Risk Springer Heidelberg.

[4] KPMG. 2014. Connected cars to deliver huge UK jobs boost, finds first UK study. http://www.kpmg.com/uk/en/issuesandinsights/arti clespublications/newsreleases/pages/connected-cars-todeliver-huge-uk-jobs-boost-finds-first-uk-study.aspx

[5] Intelligence Squared (2013) Smarter Mobility: An Evening of Debate. http://www.intelligencesquared.com/events/smartermobility-an-evening-of-debate/

[6] McBride,N ., "ACTIVE ethics: an information systems ethics for the Internet age, " Journal of Information, Communication and Ethics in Society, vol. 12, no. 1, 2014, pp. 21-43.

[7] Waldrop, M.M. 2015. Autonomous Vehicles: No Driver Required. Nature. 518 (2015), 20-23.

[8] Wilton, P. (2015) 8 Things about Oxford's driverless tech. http://www.ox.ac.uk/news/scienceblog/8-things-about-oxford $\%$ E2\%80\%99sdriverless-tech 